\title{
Susceptibility-Weighted MRI in the Evaluation of Gynecologic Diseases
}

\author{
Mohsen Kamel Arid ${ }^{*}\left(\mathbb{D}\right.$, Hamada M. Khater ${ }^{2}$ \\ ${ }^{1}$ Department of Radiology, Benha Faculty of Medicine, Benha, Egypt \\ ${ }^{2}$ Lecturer of Radiology, Faculty of Medicine, Benha University \\ Email: *mkarid2002@yahoo.com
}

How to cite this paper: Arid, M.K. and Khater, H.M. (2021) Susceptibility-Weighted MRI in the Evaluation of Gynecologic Diseases. Open Journal of Medical Imaging, 11, 6-17.

https://doi.org/10.4236/ojmi.2021.111002

Received: November 30, 2020

Accepted: February 15, 2021

Published: February 18, 2021

Copyright ( 2021 by author(s) and Scientific Research Publishing Inc. This work is licensed under the Creative Commons Attribution International License (CC BY 4.0).

http://creativecommons.org/licenses/by/4.0/ (c) (i) Open Access

\begin{abstract}
Introduction: Owing to the advanced development of MRI science, it causes obvious great changes of many diseases that affect the female genital system and affect their fertility. Hemorrhagic gynecological diseases especially endometriosis affect young females and cause cyclic pain, in addition to infertility. So early detection is essential for proper treatment. Susceptibility-weighted (SWI) as one of the most recent newly created MRI sequences is highly sensitive to detect products of hemorrhage within different gynecologic disorders with $94.7 \%$ sensitivity being more meticulous than conventional MRI sequences as T1 and T2. Aim of the Work: A comparison between T1 and T2 as conventional MRI sequences with susceptibility-weighted images (SWI) in many gynecologic disorders by the detection of the presence of internal products of hemorrhage at any stage. Subjects and Methods: 48 consecutive patients from Benha University clinics (age range, 17 - 60 years; mean age, 35.67 years). The patients included in the study were presenting with pelvic pain, irregular menses, Dyspareunia, and swelling. All with suspicious diagnosis of ovarian and extra-ovarian lesions. 38 patients out of the 48 patients were known to contain hemorrhagic disorder; all the patients underwent MRI routine pelvis protocol adding SWI sequence. Results: There was a greatly significant difference between SWI and conventional MRI sequences T1and T2 with sensitivity $94.7 \%, 57.9 \%$ and $33.3 \%$ respectively. Conclusion: SWI is a promising tool in the evaluation of hemorrhagic foci within different gynecological disorders. The great ability of detecting hemosiderin foci increases the value of SWI over conventional MRI or US.
\end{abstract}

\section{Keywords}

Endometriosis, Susceptibility Weighted Imaging (SWI), Adenomyosis, Signal Void, Magnetic Resonance Imaging, Hemorrhagic Gynecological Disease 


\section{Introduction}

Hemorrhagic gynecological diseases especially endometriosis is of most common causes of infertility that affect young females manifesting primarily as a cyclic pain as well as dysmenorrhea, so its early diagnosis sometimes becomes a challenge and is essential for proper treatment.

MRI can differentiate fine details about the composition of soft-tissue masses using differences in MR relaxation criteria seen in various types of tissue. This detail is precious in determining the characters of soft-tissue masses [1].

One of the newly created MRI functional sequences is SWI that is greatly able to detect compounds that distort the local magnetic field producing signal void. This property enables SWI to detect hemosiderin, deoxyhemoglobin, calcium, etc. So, it is the sequence that can detect even minimal hemorrhagic foci. Due to the successful use in imaging of CNS disorders, recently used in imaging the pelvis as well [2].

Different bleeding stages produce different products like methemoglobin in subacute blood products, as well as deoxyhemoglobin and hemosiderin in acute and chronic blood products respectively, all cause local magnetic field inhomogeneity in Susceptibility Weighted Image and so detected as signal drop [3].

SWI is highly sensitive for detecting products of hemorrhage within various gynecologic pathologies and helpful for the differential diagnosis such as:

Gynecological disorders associated with bleeding pathology like: hemorrhagic ovarian cysts, ovarian cancers endometriosis, hemorrhagic uterine pathologies (cancers, adenomyosis with hemorrhagic foci, leiomyomas with red degeneration, with hemorrhagic necrosis, and Placental polyp with hemorrhagic foci) are now detected more easily without the need to do extra sequences like T1-fat sat owing to the great sensitivity and specificity [3].

One of the most common challenging causes of infertility is endometriosis while its early detection helps in nearly complete and proper treatment of infertility, its late diagnosis affecting young females by repeated cyclic hemorrhage and rupture leading to adhesions leading to difficulty treated infertility. Here, we can appreciate how much the great value of SWI in early detection and proper treatment of endometriosis [4].

SWI help in diagnosing atypical cases. Because typical MRI appearance include: hyperintense T1WI cysts and/or hypointense T2WI cyst (shading). However, solid endometriomas do not meet these criteria (seen as solid masses not cysts), and it could be difficult to differentiate from other ovarian cystic masses. SWI detect it as homogenous signal void lesions [4].

Extra-ovarian endometriosis such as broad ligament, pelvic wall, caesarian section scar, urinary bladder, abdominal wall, endometriosis. etc. Could be confused with metastasis/other tumors due to their multiplicity when detected by the conventional MRI sequences (T1, T2). However, SWI can easily detect them as foci of signal voids or homogenous signal drop due to hemosiderin deposition without any confusion [2]. 
SWI is highly comparative to conventional MRI sequences showing great and significant difference in sensitivity for detecting bleeding products as SWI can detect even minimal amount of bleeding products in any stage which not the same in conventional MRI sequences [3].

Differentiating focal adenomyosis from physiologic contraction become easy task by SWI which shows adenomyosis with punctate signal voids of hemosiderin deposition scattered within adenomyosis, this not the case in contraction. [4].

Red degeneration is a hemorrhagic infarction of uterine fibroid seen during pregnancy, or with use of oral hormonal contraceptive pills that considered most sever complication. The paramagnetic methemoglobin within thrombosed veins seen as hyperintense rim on T1WI. In the other hand SWI can detect this rim at early phase as signal void due to the blooming effect of deoxyhemoglobin which cannot be done by T1WI [5].

\section{Subjects and Methods}

48 consecutive patients from Benha University clinics (age range, 17 - 60 years; mean age, 35.67 years) with different clinical presentations were enrolled in this study. Pelvic pain is the most common presentation $(43.3 \%)$, followed by Left iliac pain (13.3\%), Right iliac pain (13.3\%), pelvic pain with irregular menses (10.0\%), Dyspareunia (6.7\%), and Pelvic pain with swelling (6.7\%) (Figure 2).

All with clinical suspicious ovarian and extra-ovarian lesions.

38 patients were known to contain hemorrhagic disorder; all the patients underwent MRI routine pelvis protocol adding SWI sequence. They have been evaluated at the Radiology Department, faculty of medicine, Benha University Hospitals.

We include patients with Hemorrhagic ovarian cysts, Endometriosis, ovarian cancers, cancer cervix, extraovarian endometriotic implants (peritoneal. scar, anterior abdominal wall), Hemorrhagic Uterine pathologies (Adenomyosis with hemorrhagic foci, and Leiomyomas with red degeneration and hemorrhagic necrosis). All patients enrolled in the study, have normal renal function tests (Table 2).

We exclude patients with ferromagnetic or electronically operated active devices like automatic cardioverter defibrillators, cardiac pacemakers, cochlear implants and Patients with high renal function tests.

Examinations were performed regardless of the stage of the menstrual cycle with no bowel preparation or intraluminal opacification of bowel or vagina will be used.

Images was acquired on a $1.5 \mathrm{~T} \mathrm{MR}$ scanner equipped with an 8-channel pelvic coil. Standard pelvic MRI protocol including three projections: axial, sagittal, and coronal T2- and T1-pulse sequences before and after fat-suppression and gadolinium IV contrast.

The additional time for SWI sequence is approximately $3 \mathrm{~min}$. SWI sequences 
consisting of magnitude and phase images will be obtained in axial planes.

All images were reviewed on PACS (picturing archiving and communication system) workkstation and lesions were evaluated regarding their location, signal intensity on T1W and T2W images, and presence of signal void on SWI. The lesion signal intensity will be considered hypointense or hyperintense compared to the signal intensity of adjacent pelvic muscles.

\section{Results}

Table 1: This table shows the patient ranged age 17 - 60 with mean $35.67 \pm 11.16$ and median 36.50 (16.5).

Figure 1: Histogram distribution of gynecologic diseases according to their age.

Figure 2: Shows clinical presentations of the cases: Pelvic pain (43.3\%), Left iliac pain (13.3\%), Right iliac pain (13.3\%), Pelvic pain and Irregular menses (10.0\%), Dyspareunia (6.7\%), Pelvic pain and swelling (6.7\%), Abdominal and suprapubic pain (3.3\%) and Pelvic pain and dyspareunia (3.3\%) of complaint.

Table 1. Demographic characteristics of patients.

\begin{tabular}{cc}
\hline Age (years) & Statistics \\
Range & $17-60$ \\
Mean \pm SD & $35.67 \pm 11.16$ \\
Median $(\mathrm{IQR})^{*}$ & $36.50(16.5)$
\end{tabular}

*Interquartile range.



Figure 1. Histogram distribution of gynecologic diseases according to their age. 
Table 2: Distribution of gynecologic diseases according to their findings: Endometrioma (20.8\%), Hemorrhagic cysts (16.66\%), Endometriotic implant (10.41\%), Interstitial fibroid (10.41\%), Multiloculated cysts (10.41\%), Adenomyosis (6.25\%), Complicated ovarian cyst (6.25\%), Endometriotic cysts (6.25\%), Simple Ovarian cysts (6.25\%), Ovarian mass contain hemorrhagic cyst (4.1\%) and Subserous fibroid with hemorrhagic foci (2\%) of Findings.

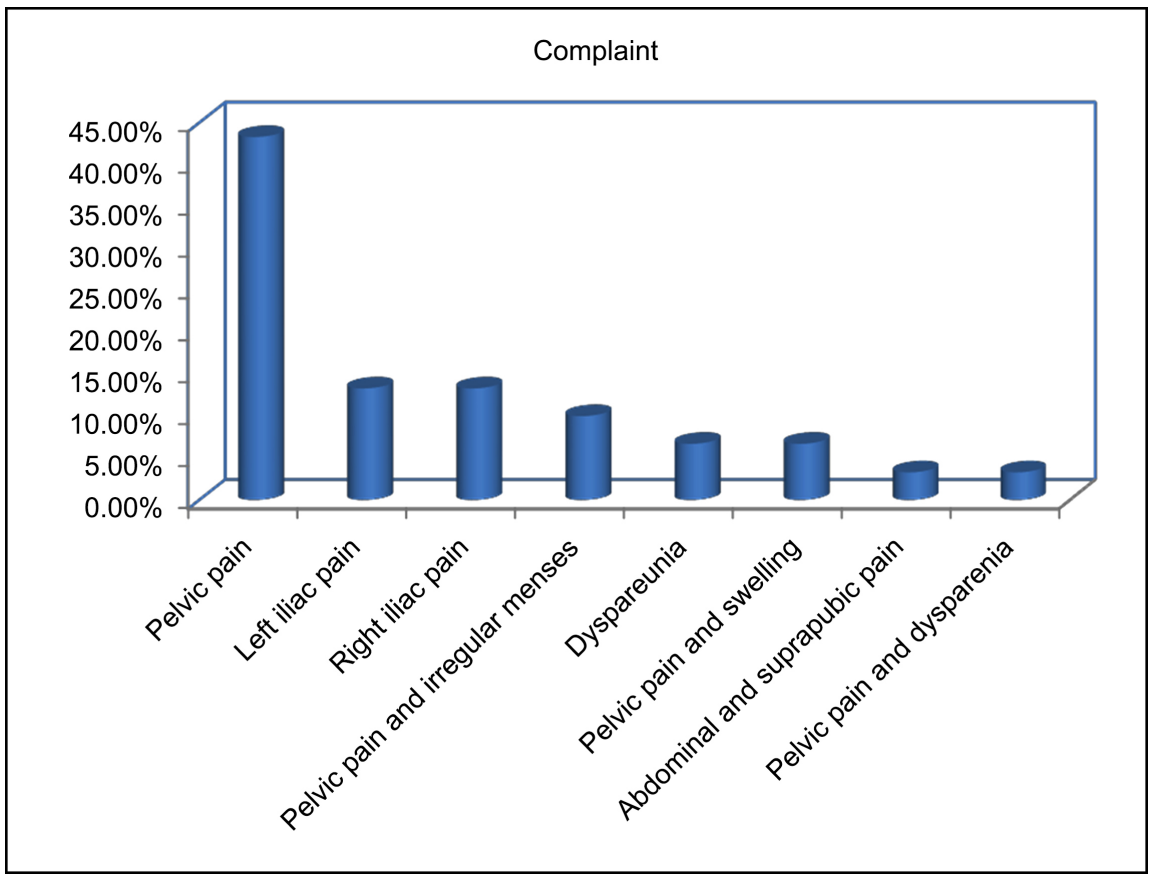

Figure 2. Distribution of clinical presentation.

Table 2. Distribution of gynecologic diseases according to their findings $(n=48)$.

\begin{tabular}{ccc}
\hline Findings & No. & $\%$ \\
\hline Endometrioma & 10 & $20.8 \%$ \\
Hemorrhagic cysts & 8 & $16.66 \%$ \\
Endometriotic implant & 5 & $10.41 \%$ \\
Interstitial fibroid & 5 & $10.41 \%$ \\
Multiloculated cysts & 5 & $10.41 \%$ \\
Adenomyosis & 3 & $6.25 \%$ \\
Complicated ovarian cyst & 3 & $6.25 \%$ \\
Endometriotic cysts & 3 & $6.25 \%$ \\
Simple Ovarian cysts & 3 & $6.25 \%$ \\
Ovarian mass contain hemorrhagic cyst & 2 & $4.2 \%$ \\
Subserous fibroid with hemorrhagic foci & 1 & $2.1 \%$ \\
Total & 48 & $100.0 \%$ \\
\hline
\end{tabular}


Table 3: Distribution of gynecologic diseases according to their T1, T2, SWI and known cases with hemorrhage shows positive T1 signal intensity (50\%), T2 signal intensity (33.3\%), SWI (75\%) by MRI and Known cases with hemorrhage (79.1\%).

Figure 3: Shows highly statistically significant agreement between SWI and known cases with hemorrhage among studied group.

Figure 4: Diagnostic performance of known cases with hemorrhage in reference to T1 signal intensity by MRI, Sensitivity $57.9 \%$, specificity $80.0 \%$, positive predictive value $91.6 \%$, negative predictive value $33.3 \%$ and accuracy $62.5 \%$.

Figure 5: Diagnostic performance of known cases with hemorrhage in reference to SWI, sensitivity $94.7 \%$, specificity $100.0 \%$, positive predictive value $100.0 \%$, negative predictive value $83.3 \%$ and accuracy $=95.8 \%$.

Table 3. Distribution of gynecologic diseases according to T1 and T2, SWI in addition to the known cases with hemorrhage $(n=48)$.

\begin{tabular}{ccc}
\hline MRI & Negative & Positive \\
\hline T1 SI & $24(50 \%)$ & $24(50 \%)$ \\
T2 SI & $32(66.7 \%)$ & $16(33.3 \%)$ \\
SWI & $12(25 \%)$ & $36(75 \%)$ \\
Known cases with hemorrhage & $10(20.83 \%)$ & $38(79.17 \%)$ \\
\hline
\end{tabular}

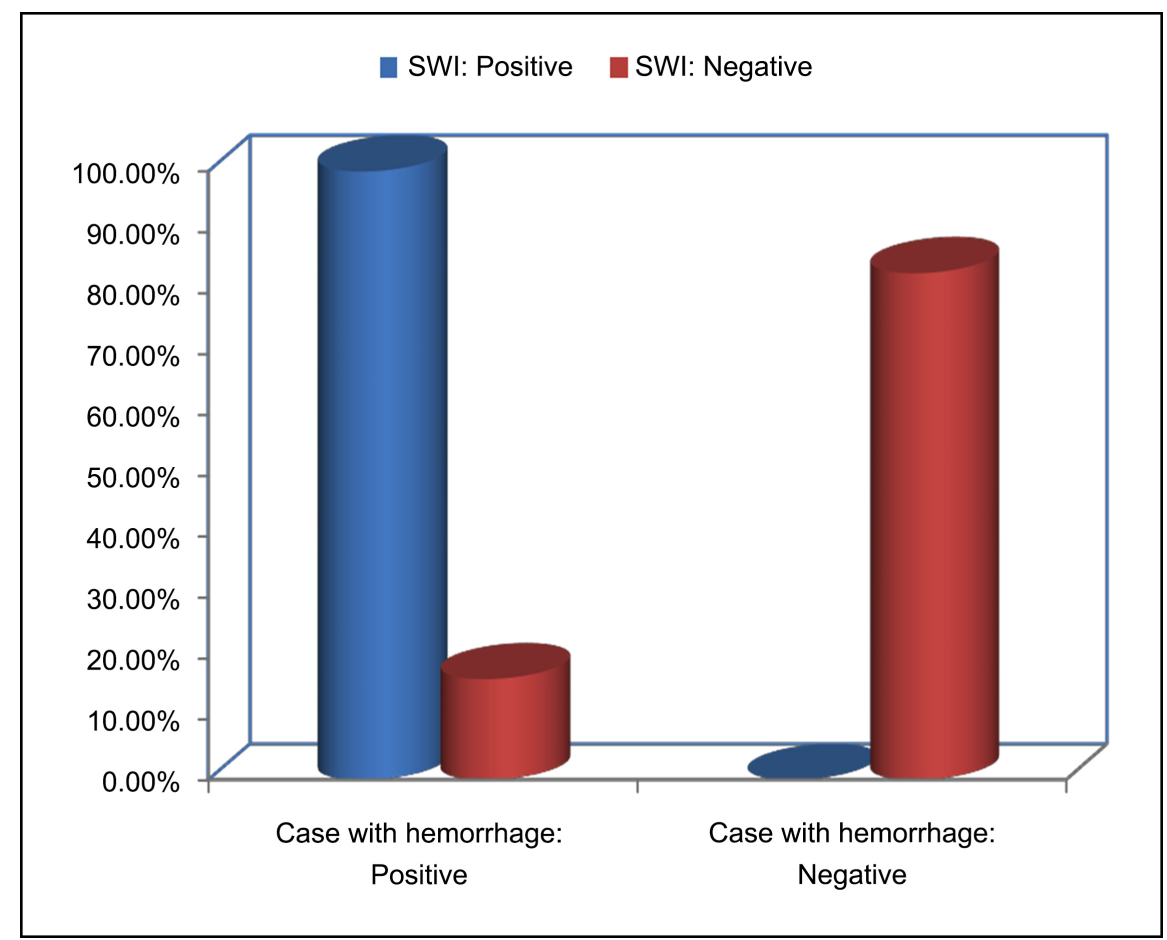

Figure 3. Agreement between SWI and known cases with hemorrhagic pathology among studied group. 


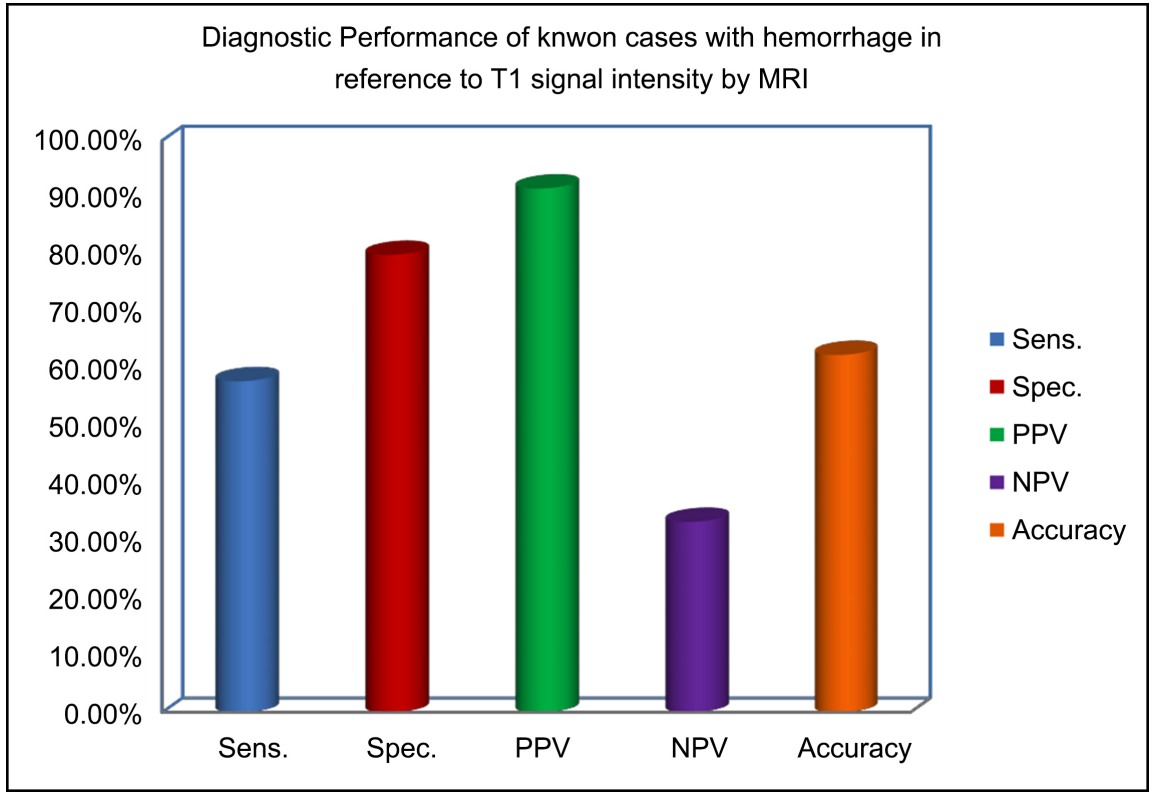

Figure 4. Diagnostic performance of known cases with hemorrhage in reference to T1 signal intensity by MRI.

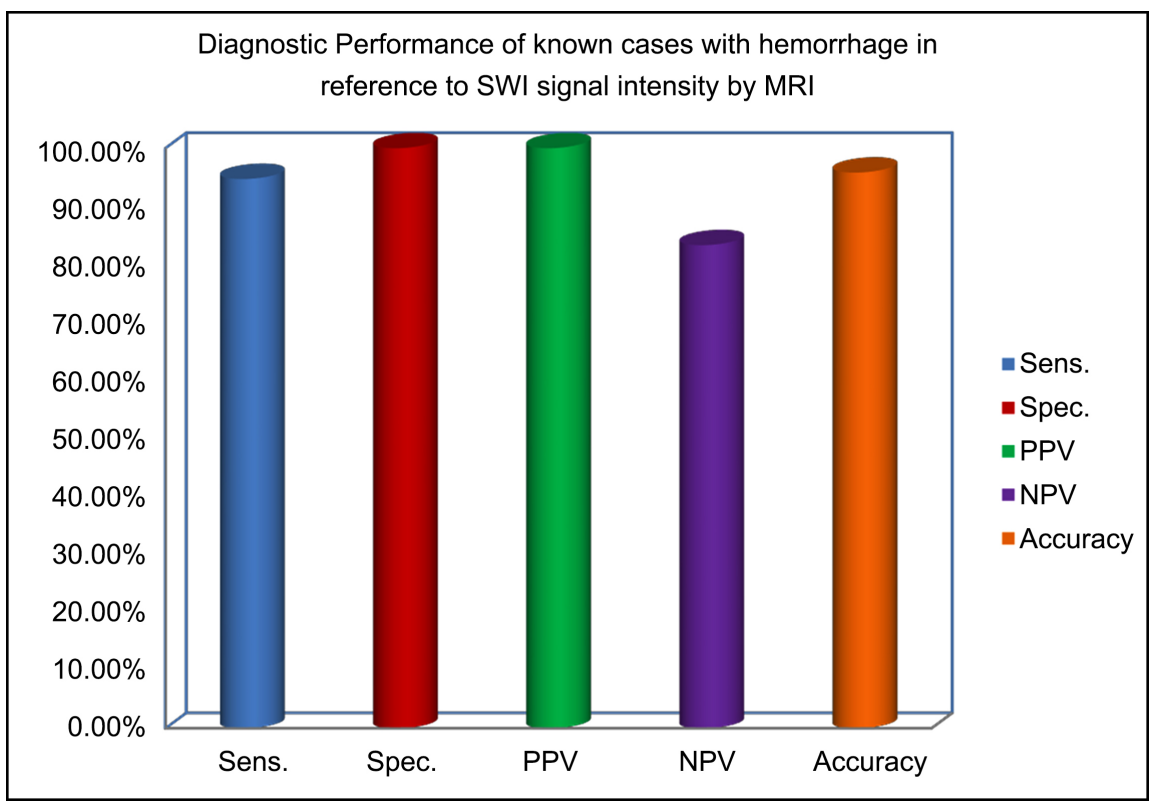

Figure 5. Bar chart diagnostic performance of known cases with hemorrhage in reference to SWI signal intensity by MRI.

\section{Case Presentations}

Case 1, Figure 6 \& Figure 7: a 26-year-old female patient complaining of pelvic pain. Ultrasound showed bilateral ovarian cysts for MRI assessment, and the final diagnosis was bilateral endometriomas.

Case 2, Figure 8 \& Figure 9: a 50-year-old female patient with of pelvic pain and mass sensation, ultrasound examination shows bilateral ovarian cysts, and the final diagnosis was bilateral endometriomas. 
Case 3, Figure 10 \& Figure 11: a 53-year-old female patient with recurrent vaginal bleeding, and the final diagnosis was cancer cervix with scattered foci of microbleeds.

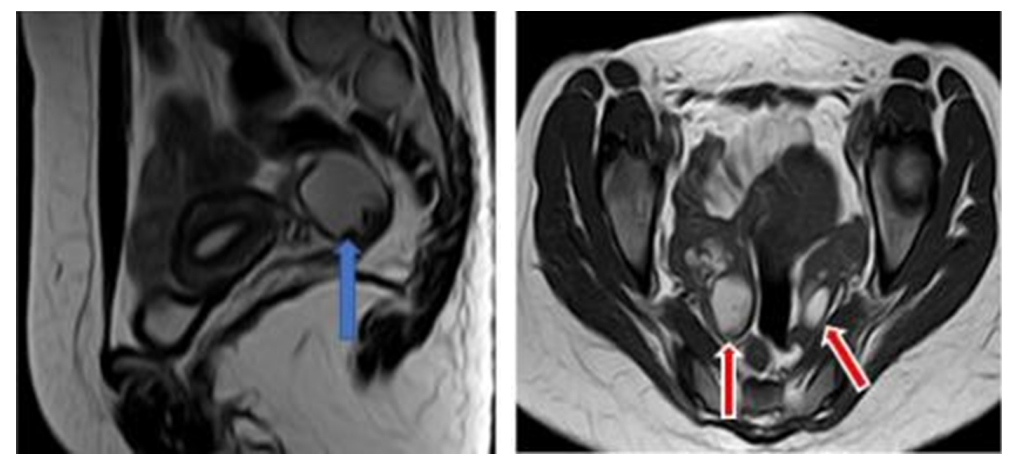

Figure 6. Left image is sagittal T2 WIs: behind the uterus one ovary shows a small cyst with low signal intensity (blue arrow). Right image, Axial T1 WIs: showing bilateral ovarian cysts with high signal content (red arrows).

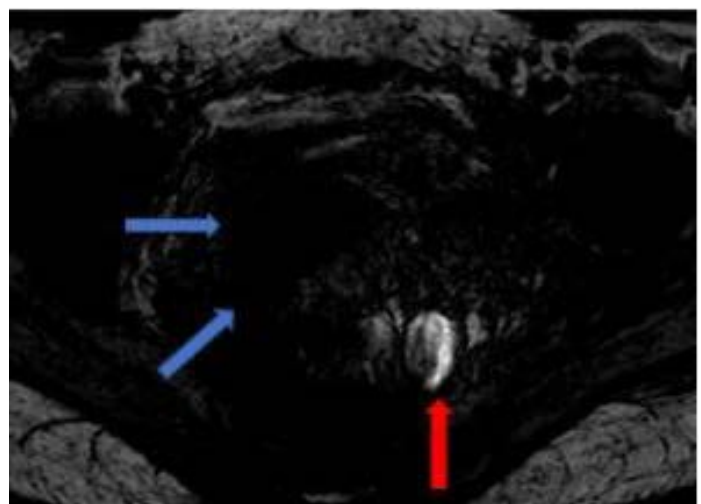

Figure 7. Axial SWI: complete signal loss/void at cyst of right ovary (blue arrows). The left ovarian cyst shows high signal with intervening areas of low signal (red arrow).

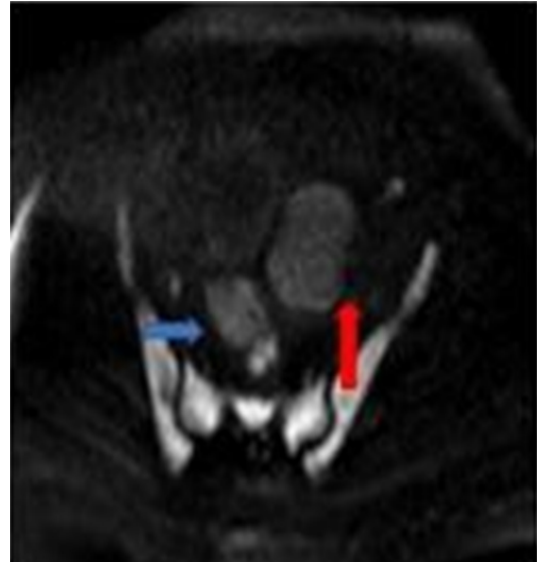

(a)

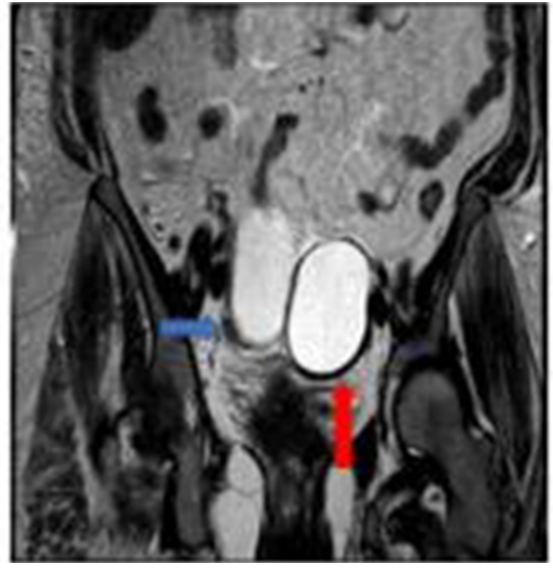

(b)

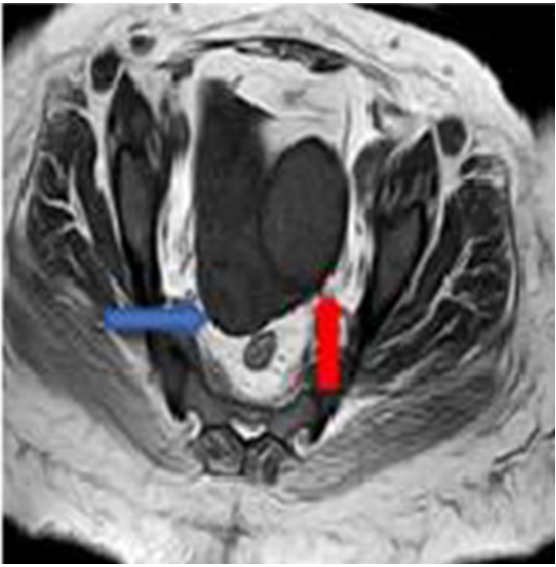

(c)

Figure 8. An axial diffusion weighted (DWI) shows no restriction of both ovarian cysts (arrows); (b) coronal T2WIs showing bilateral ovarian cysts with bright homogenous fluid content (arrows); (c) axial T1WI: ovarian cyst on both sides show intermediate signal (arrows). 


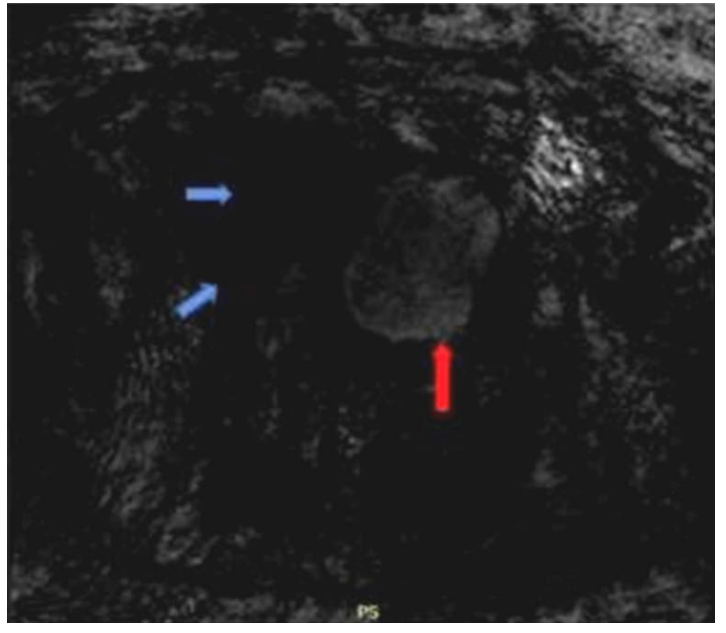

Figure 9. Axial SWI: right ovarian cyst is of homogenously low signal intensity (blue arrows) and left ovarian cyst is of mixed low and high signal intensity mostly low (red arrow).

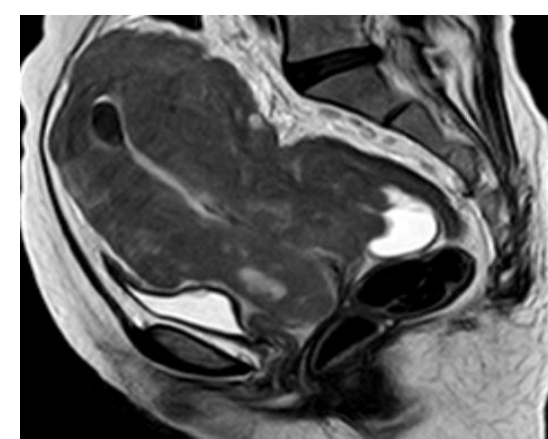

(a)

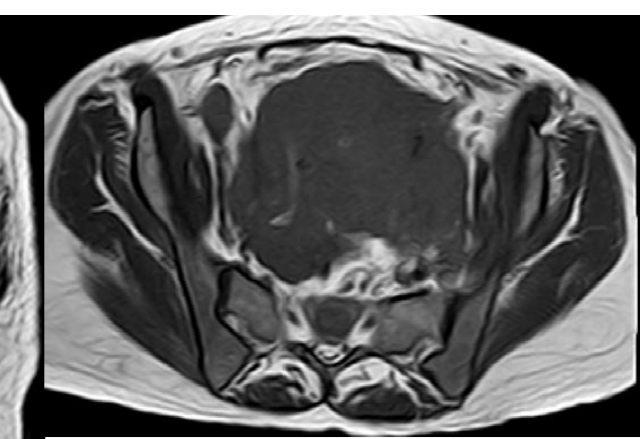

(b)

Figure 10. (a) Coronal T2WIs displays non homogenous signal intensity with areas of high signal at the cervical region associated with endometrial thickening; (b) Axial T1WI: show huge irregular cervical mass lesion being of low signal intensity interlaced with foci of high signal.

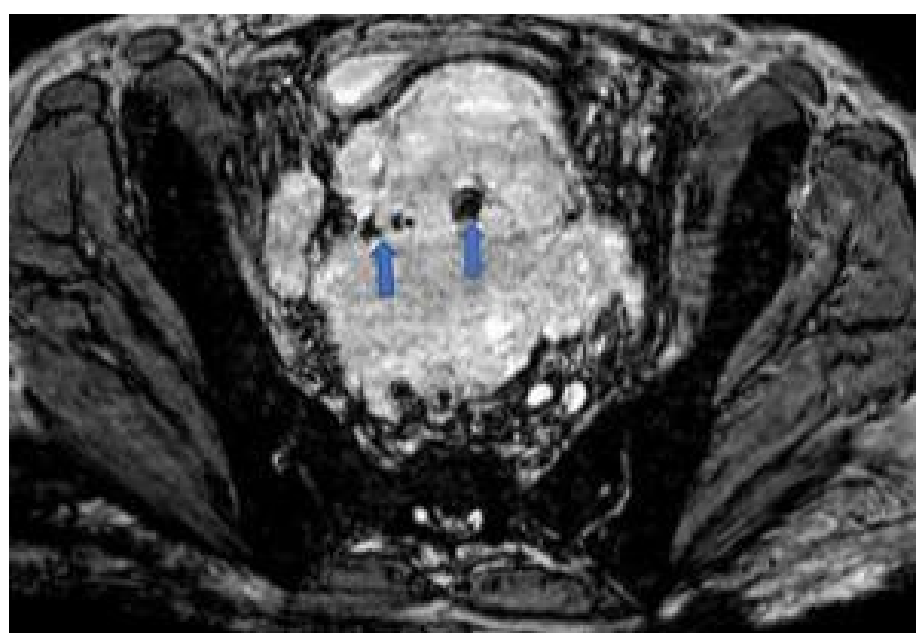

Figure 11. Axial SWI: showing multiple small foci of signal void at the cervical mass lesion denoting hemorrhage (blue arrows). 


\section{Discussion}

SWI is superior to conventional MRI sequences T1, T2 weighted images as SWI is greatly sensitive for showing blood products and venous vasculature being complementary to conventional sequences. Good pathologic correlations were found for blood products as predicted by SWI [6].

The current study was done to highlight the diagnostic value of SWI in various gynecological diseases that contain hemorrhagic products in comparison to conventional sequences ( $\mathrm{T} 1$ and $\mathrm{T} 2 \mathrm{WIs}$ ).

48 patients of ( 17 - 60 y.o) age groups were tested with ovarian and extra-ovarian hemorrhagic diseases suspected clinically and by US. 38 cases of them were known to have hemorrhage e.g. Endometrioma, adenomyosis, hemorrhagic cyst, gynecological tumor, etc.

From the 48 cases we examined. 24 cases (50 \%) showed hyperintense T1, 10 cases showed hypointense $\mathrm{T} 1$ signal, 10 cases of intermediate $\mathrm{T} 1$ signal and 4 cases showed heterogenous T1 signal. 4 cases showed intermediate T2 SI, 4 cases of low T2 SI, 24 cases showed T2 hyperintense signal and 16 cases showed a shading sign in T2 WI. 36 cases showed blooming/signal void in the SWI sequence, 2 cases of heterogenous signal and only 10 cases showed high signal in SWI.

These results agreed with Sakshi, et al. (2016) [7], who concluded that the MRI picture of the endometrioma was found to be almost typical in all cases. T1WI showed hyperintense signals, not suppressed in fat suppression sequences. T2WI showed hypointense signals (shading). SWI showed signal void (blooming) in all cases (100\%).

Also agreed with the study by Dogheim, et al. (2014) [8] that showed 11 cases (100\%) of endometriomas that displayed blooming in SWI.

Out of the 38 cases known to have hemorrhage of our study, 24 cases showed high signal in T1WI without suppression in T1fs as well as 16 cases showed T2 shading sign denoting hemorrhagic products, giving $57.9 \%$ and $33.3 \%$ sensitivity respectively as well as $62.5 \%$ and $44.2 \%$ accuracy respectively. In the other hand 36 cases show signal void in SWI denoting hemorrhagic products giving 94.7\% sensitivity and $95.8 \%$ accuracy.

These results agreed with Takeuchi Mayumi (2008) [1] in susceptibility-weighted MR images, punctate or curved linear signal voids along the cyst wall were observed in 39 endometriomas $(92.9 \%)$ and in no non endometrial cysts. Thirty-two endometriomas (76.2\%) met definitive MRI criteria, that is, hyperintensity on T1-weighted images and hypointensity on T2-weighted images, and 41 endometriomas (97.6\%) were correctly diagnosed with susceptibility-weighted MRI.

Also agreed with Dogheim, et al. (2014) [8] who processed their study upon 25 cases of different gynecological disease with 11 cases of them known to be diagnosed as endometrioma. Out of 11 cases of known diagnosis as endometrioma, 9 cases (81.8\%) showed high T1 signal intensity, on the other hand 11 cases 
(100\%) show blooming in SWI sequence.

\section{Conclusion}

SWI is an excellent MRI sequence showing great sensitivity and accuracy in the evaluation of different pathologies related to the gynecologic diseases that contain hemorrhagic products. SWI is superior to conventional MRI in assessing hemosiderin deposition which makes it of more value than conventional MRI or Ultrasonography.

\section{Ethics Statement}

The study was approved by the Ethics Board of Benha University.

\section{Conflicts of Interest}

The authors declare no conflicts of interest regarding the publication of this paper.

\section{References}

[1] Takeuchi, M., Matsuzaki, K. and Nishitani, H. (2008) Susceptibility-Weighted MRI of Endometrioma: Preliminary Results. American Journal of Roentgenology, 191, 1366-1370. https://doi.org/10.2214/AJR.07.3974

[2] Deistung, A. (2008) Susceptibility Weighted Imaging at Ultra High Magnetic Field Strengths: Theoretical Considerations and Experimental Results. Magnetic Resonance in Medicine, 60, 1155-1168. https://doi.org/10.1002/mrm.21754

[3] Krishnan, A.S., Lansley, J.A., Jäger, H.R. and Mankad, K. (2015) New Vistas in Clinical Practice: Susceptibility-Weighted Imaging. Quantitative Imaging in Medicine and Surgery, 5, 448-452.

[4] Takeuchi, M., Matsuzaki, K. and Harada, M. (2015) Susceptibility-Weighted MRI of Extra-Ovarian Endometriosis: Preliminary Results. Abdomn Imaging, 40, 2512-2416. https://doi.org/10.1007/s00261-015-0378-Z

[5] Wu, Z., Mittal, S. and Kish, K. (2009) Identification of Calcification with MRI Using Susceptibility-Weighted Imaging: A Case Study. Journal of Magnetic Resonance Imaging, 29, 177-182. https://doi.org/10.1002/jmri.21617

[6] Sehgal, V., Delproposto, Z., Haddar, D., et al. (2006) Susceptibility-Weighted Imaging to Visualize Blood Products and Improve Tumor Contrast in the Study of Brain Masses. Journal of Magnetic Resonance Imaging. An Official Journal of the International Society for Magnetic Resonance in Medicine, 24, 41-51. https://doi.org/10.1002/jmri.20598

[7] Sakshi, K.H., Prativa, S., Mukta, K., et al. (2016) Susceptibility Weighed with Quantitative Phase Magnetic Resonance Imaging in Differentiation of Different Stages of Hemorrhage and Calcification in Female Pelvic Pathologies. A Preliminary Study. Journal of Computer Assisted Tomography, 41, 586-591. https://doi.org/10.1097/RCT.0000000000000551

[8] Dogheim, O.Y., Abdel Hamid, A.M., Barakat, M.S., Eid, M. and El-Sayed, S.M. (2014) Role of Novel Magnetic Resonance Imaging Sequences in Characterization of Ovarian Masses. The Egyptian Journal of Radiology and Nuclear Medicine, 45, 237-251. https://doi.org/10.1016/j.ejrnm.2013.11.008 


\section{Abbreviation}

MRI: magnetic resonance imaging,

US: ultrasound,

SWI: susceptibility weighted,

DWI: diffusion weighted,

T1 fs: T1 fat suppression. 